\title{
Altered regulation of Toll-like receptor responses impairs antibacterial immunity in the allergic lung
}

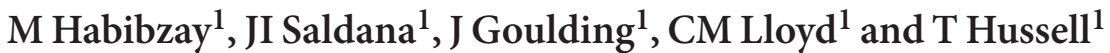

The lung is colonized by commensal bacteria, some of which are associated with asthma exacerbations. Using the intranasal house-dust mite-sensitized mouse model of allergic airway disease, we show an imbalance in novel antibacterial pathways that culminates in a reduction in neutrophil recruitment to the airspaces and leads to bacterial invasion and dissemination. The expression of TREM (Triggering Receptor Expressed on Myeloid cells)-1 that amplifies Toll-like receptor (TLR) signaling and TREM-2 that inhibits this process is reversed. Furthermore, endogenous TLR inhibitors (A20, Tollip, SOCS1, and IRAK-M) and proteins involved in receptor recycling (TRIAD3) are raised.

Consequently, the production of neutrophil chemoattractants is reduced. Intranasal administration of either chemokine restores the ability to recruit neutrophils, which prevents bacterial invasion. A background of allergic airway disease therefore exacerbates bacterial infection by altering key antibacterial innate immune pathways that are amenable to therapeutic intervention.

\section{INTRODUCTION}

Allergic airway disease and bacterial infection independently affect millions of people world-wide, causing significant mortality and an associated high economic burden. Streptococcus pneumoniae (S. pneumoniae or pneumococcus) is a Grampositive bacterium commonly found as a commensal of the nasopharynx but is the leading cause of morbidity and mortality among children, ${ }^{1}$ being more prevalent in asthmatics. ${ }^{2,3}$ The outcome of infection is most commonly pneumococcal pneumonia, but meningitis, sepsis, and otitis media are also prevalent. Once invasive, the prognosis is poor, leading to bacteremia and meningitis. Complications due to lung pneumococcal infection usually occur in a background of other conditions, including underlying chronic disease, a disruption of the lung microenvironment by viral infection, or immune deficiencies. S. pneumoniae, Haemophilus influenzae, and Moraxella catarrhalis are also found during exacerbation of asthma, ${ }^{4}$ and the response of asthmatics to antibiotics suggests the importance of acute and chronic bacterial infections in the pathogenesis of disease. ${ }^{5}$

Signaling via Toll-like receptor (TLR)-2, 4, and 9 (recognizing bacterial lipoteichoic acid, pneumolysin, and bacterial DNA, respectively) is important for initiation of innate inflammation and recruitment ${ }^{6}$ via release of interleukin
(IL)-12, IL-17A, IL-18, IL-6, IL-8 and especially tumor necrosis factor- $\alpha$ and IL-1 to a pneumococcal infection. These cytokines are central to neutrophil recruitment, intracellular killing of bacteria via nuclear factor- $\kappa B$ RelA activation, ${ }^{7}$ and the development of adaptive immunity, including neutrophilrecruiting Th17 cells (for reviews, see Paterson and Orihuela, ${ }^{6}$ Malley, ${ }^{8}$ and Kolls $\left.{ }^{9}\right)$. However, the Th2 cytokine-dominated environment of the allergen-exposed lung may impede activation of appropriate innate pathways. For example, IL-33 is highly expressed in the asthmatic lung, but upon binding its receptor ST2L the TLR adaptor protein MyD88 is recruited ${ }^{10}$ and may therefore compete with pathways promoting antibacterial immunity.

We hypothesized that the allergen-exposed lung is unable to mount sufficient antipneumococcal responses and aimed to define the altered molecular mechanisms leading to this in the murine model of house-dust mite (HDM)-induced allergic airway disease. Also, $50-85 \%$ of asthmatics are HDM allergic, and it is therefore a clinically relevant allergen to use, that, unlike ovalbumin, does not require intraperitoneal priming. ${ }^{11}$ We show that $S$. pneumoniae becomes fatal following airway exposure to HDM owing to impeded recruitment of neutrophils caused by (i) a desensitization of TLRs, (ii) an upregulation of endogenous negative regulators of TLRs (A20, IRAK-M, TOLLIP),

\footnotetext{
${ }_{1}^{1}$ Imperial College London, Leukocyte Biology Section, National Heart and Lung Institute, London, UK. Correspondence: T Hussell (t.hussell@imperial.ac.uk and t.hussell@ic.ac.uk)

Received 16 January 2012; accepted 19 March 2012; published online 2 May 2012. doi:10.1038/mi.2012.28
} 
(iii) a preference for TREM (Triggering Receptor Expressed on Myeloid cells)-2 expression that inhibits TLR signaling rather than TREM-1 that promotes, and ultimately (iv) a reduction in neutrophil chemoattractants. Furthermore, despite the complexity of antibacterial pathways that are disrupted, the therapeutic re-introduction of a single chemokine to mice exposed to HDM allergen enables clearance of S. pneumoniae that would otherwise prove fatal. Knowledge of antibacterial pathways disrupted in allergic airway disease provides avenues for therapeutic manipulation and more focused management of patients with this condition.

\section{RESULTS}

The protocol used for development of allergic airway disease is shown in Supplementary Figure S1 online in the online data supplement, with bacteria administered intranasally (i.n.) $72 \mathrm{~h}$ after the last HDM exposure. The dose of bacteria used causes a self-limiting infection in $\mathrm{BALB} / \mathrm{c}$ mice that is cleared within $48 \mathrm{~h}$ without any visible signs of clinical illness. In allergic mice, we first ascertained that the standard hallmarks of airway disease were present by sampling mice before bacterial challenge. Intranasal administration of HDM three times a week for 3 weeks resulted in increased mucus production (seen in histology by periodic acid-Schiff staining; see Supplementary Figure S1B online), and increased airway and lung cellularity (see Supplementary Figure S1C online) and eosinophils (see Supplementary Figure S1D online) as described before. ${ }^{12}$ S. pneumoniae administered into this environment (Figure 1a) persisted in the airway and lung, and even disseminated to the blood, but was undetectable in any compartment at $48 \mathrm{~h}$ in phosphate-buffered saline (PBS)/S. pneumoniae control mice (Figure 1b). Enhanced mucus production was once again observed in HDM-sensitized mice before bacterial infection, which became even more evident following infection (Figure 1c,d and see Supplementary Figure S2 online). The absence of mucus production in $\mathrm{PBS} / \mathrm{S}$. pneumoniae mice suggests that bacteria enhance a pathological feature that is already present.

Enhanced airway (Figure 1e) and lung (Figure 1g) cellularity is observed in the HDM-sensitized mice before bacterial infection compared with PBS controls, as expected (denoted by the zero-hour time point). Cellular expansion from this baseline occurs in the PBS-sensitized mice following S. pneumoniae challenge. However, the cellularity of the HDM-sensitized airway and lung either reduces or remains the same following S. pneumoniae infection, suggesting a blunted response to this infectious organism. This is more clearly seen when the change in cellularity in the airway (Figure 1f) and lung (Figure $\mathbf{1 h}$ ) is expressed as a percentage of the baseline at day $0(72 \mathrm{~h}$ following cessation of HDM or PBS sensitization). Once again, cellularity to $S$. pneumonia increases in PBS- but not in HDMsensitized mice. In the airspaces, cellularity from baseline is even reduced by $S$. pneumoniae infection (Figure 1f), which can be explained by a reduction in eosinophils and $\mathrm{CD} 4^{+}$ $\mathrm{T}$ cells in both the airway and lung (see Supplementary Figure $\mathbf{S} 3$ online).
HDM exposure reduces recruitment of TLR-expressing neutrophils to the airway and lung without affecting IL-17A

Neutrophils are an important cell type in antibacterial immunity. We next examined whether their recruitment was affected by prior HDM exposure. Upon bacterial infection, the rapid neutrophil accumulation seen in the airspaces and lung of PBS control mice was blunted in previously HDM-sensitized mice (Figure 2a,b). The reduced airway neutrophil count was not due to a defect in their release from the bone marrow into the blood (see Supplementary Figure S4 online), but was associated with reduced levels of soluble tumor necrosis factor, IL-6, CXCL1 (KC), macrophage inflammatory protein (MIP)-2, and matrix metalloproteinase 9 (MMP-9) in airway lavage fluid (Figure $\mathbf{2 c - g}$ ). The maximum levels of these cytokines in the HDM group alone are shown at the day 0 time point, after which they reduced to negligible levels. Though total protein levels of KC were similar at $4 \mathrm{~h}$ following $S$. pneumoniae infection, analysis of the epithelium by immunohistochemistry revealed a reduction in epithelial cells from $\mathrm{HDM}$-sensitized mice (Figure 2h), whereas KC production in PBS-sensitized mice is clearly seen in epithelial cells (Figure $\mathbf{2 h}$, arrow). Of the neutrophils (Figure 3a,b) and resident $\mathrm{CD} 11 \mathrm{c}^{+}$alveolar macrophages (Figure 3c,d) recruited to or remaining in the HDM-sensitized airway, fewer of them expressed TLR-2, which is responsible for recognizing $S$. pneumoniae lipoteichoic acid (see Supplementary Figure $\mathbf{5}$ online).

IL-17A is an important stimulus for granulocyte-colonystimulating factor secretion, which is essential for production of neutrophils. Furthermore, endothelial cells activated by IL-17A can release KC and MIP-2 that activate and recruit neutrophils. The major IL-17A-producing cells are $\gamma \delta$ $\mathrm{T}$ cells, CD $4{ }^{+} \mathrm{Th} 17$, and natural killer T cells. ${ }^{13}$ As reported previously, ${ }^{14} \mathrm{IL}-17 \mathrm{~A}$-producing $\mathrm{CD} 4^{+}$and $\gamma \delta$ TCR-expressing $\mathrm{T}$ cells were elevated in the HDM-sensitized airway compared with PBS/S. pneumoniae controls (see Supplementary Figure $\mathbf{S 6}$ online). Forty-eight hours after bacterial infection, the numbers of these cells were indistinguishable from control PBS-sensitized mice or mice sensitized with HDM but without bacterial infection. Furthermore, we did not observe any significant difference in IL-17A concentration in bronchoalveolar lavage fluid (see Supplementary Figure S6 online). The difference in neutrophil recruitment between control and HDM-sensitized mice following S. pneumoniae infection cannot, therefore, be due to defective IL-17 A production. Furthermore, we did not observe any differences in antimicrobial peptides in airway lavage and only a partial decrease in C-reactive protein in HDM-sensitized mice (data not shown and see Supplementary Figure S7 online).

\section{HDM exposure reduces pathways that promote, and increases pathways that inhibit, TLR responses}

TREM-1 is expressed on neutrophils and monocytes and serves as a critical amplifier of inflammatory signaling via TLRs. ${ }^{15}$ The reduced production of inflammatory cytokines may relate to the significantly lower expression of TREM-1 we observed on neutrophils (Figure $3 \mathbf{e}, \mathbf{f}$ ) $78 \mathrm{~h}$ after the last HDM, compared 

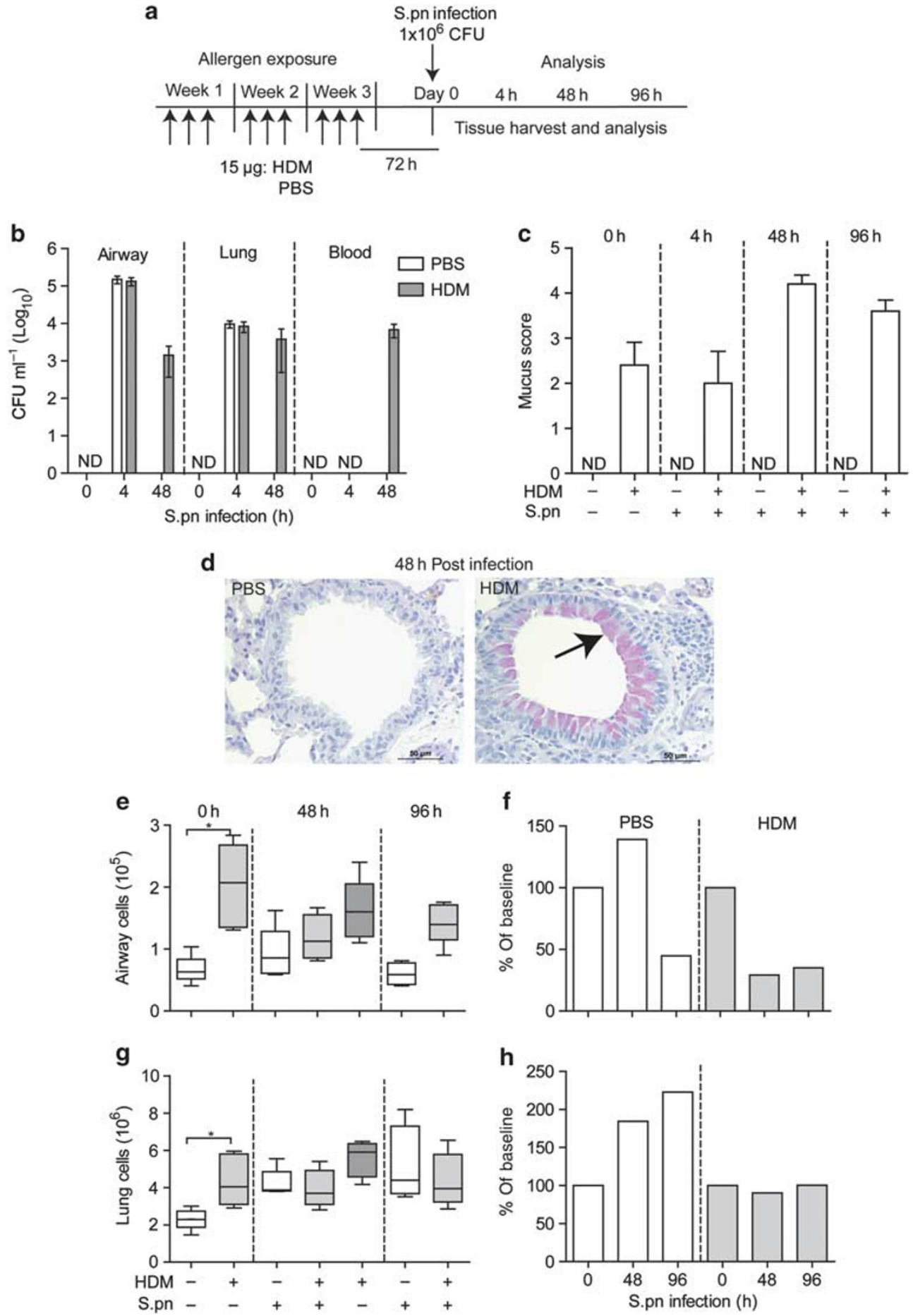

Figure 1 Allergic airway inflammation induces exacerbation of lung Streptococcus pneumoniae (S.pn) infection. (a) Experimental setup of housedust mite (HDM)-induced allergic airway inflammation and bacterial (S.pn) infection in BALB/c mice. BALB/c mice were inoculated intranasally with $15 \mu \mathrm{g}$ of HDM extract in $15 \mu \mathrm{l}$ phosphate-buffered saline (PBS) or $15 \mu \mathrm{l}$ PBS alone three times a week for 3 weeks and subsequently infected with $1 \times 10^{6} \mathrm{CFU}$ (colony-forming units) of S.pn (D39 strain) harvested on day 0 ( $72 \mathrm{~h}$ post final HDM exposure). (b) CFUs were calculated using serial dilutions of single-cell suspensions of each sample and plated on blood agar plates. Bacterial titers were measured at 0,4 , and $48 \mathrm{~h}$ post infection in bronchoalveolar lavage fluid (BALF/airway), lung, and blood. (c) Mucus scoring show the presence of enhanced mucus production observed in HDM-sensitized mice before bacterial infection, which became even more evident following infection. (d) Periodic acid-Schiff -stained lung sections showing increased mucus production by goblet cells (arrows) $48 \mathrm{~h}$ post infection in the PBS- and HDM-exposed groups and analyzed by light microscopy. Pictures show representative samples of five mice per group from one experiment and are representative of two independent experiments. (e) Airway and (g) lung total viable cell numbers. (f) Airway and (h) lung cell counts presented as percentage of baseline ( $0 \mathrm{~h}$ after infection). Data presented as box and whisker plots, with a median of $n=13-15$ per group. ${ }^{*} P<0.05$. ND, not detected. 
a

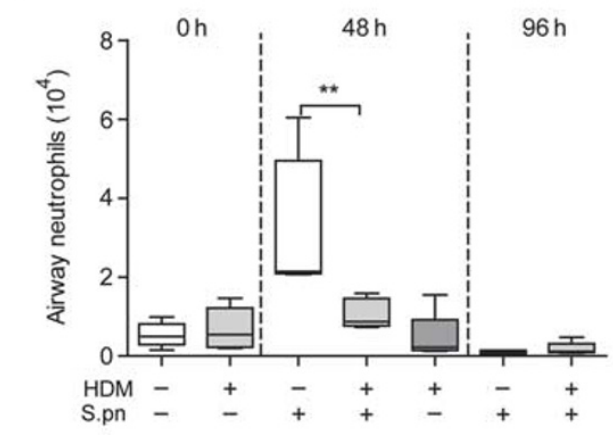

b

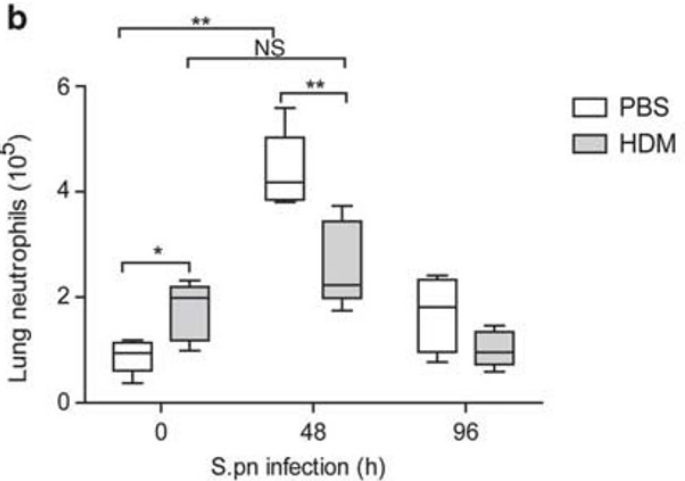

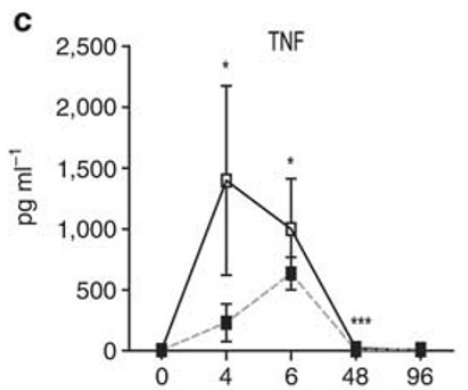
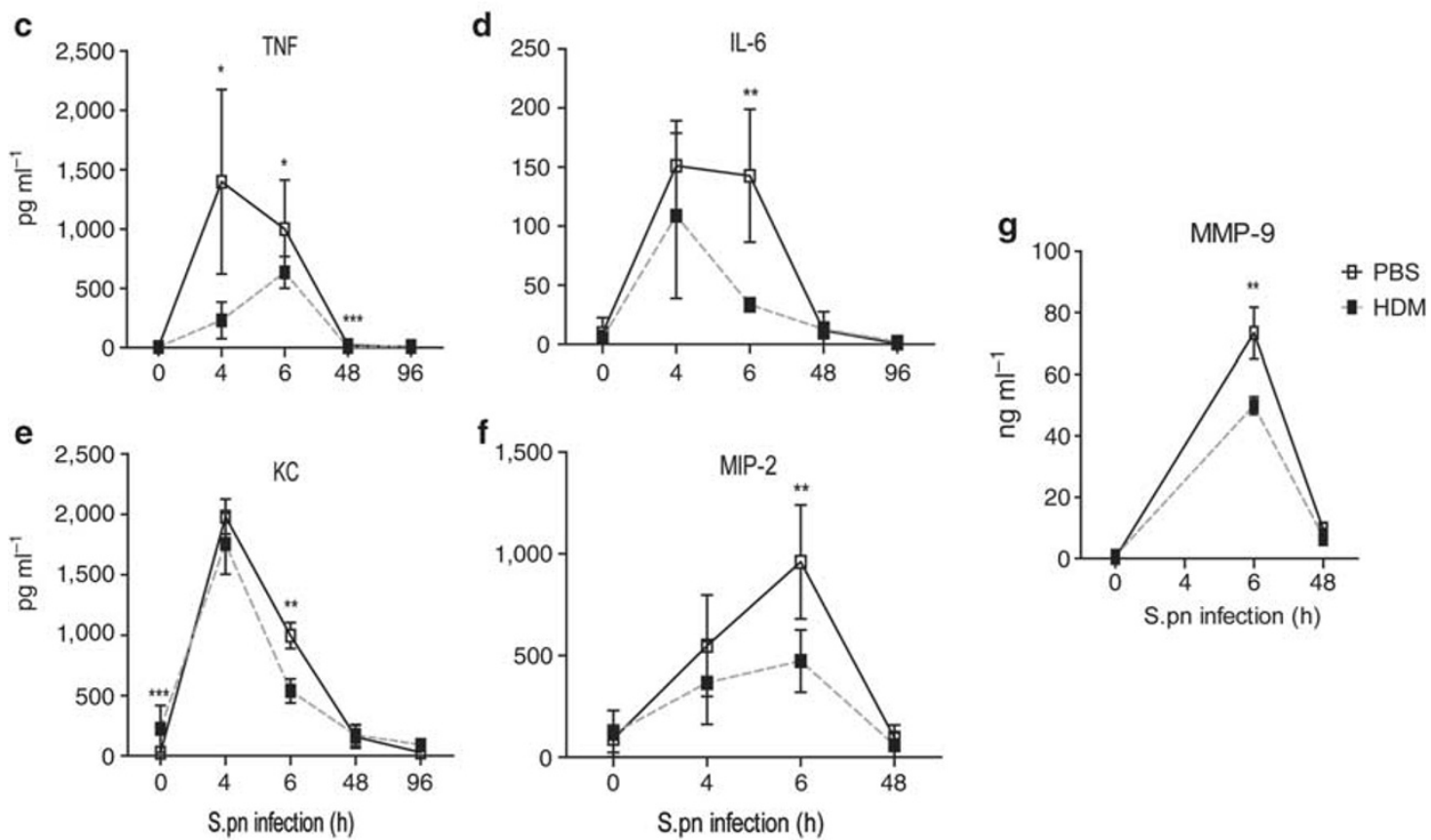

h

4 Hours after S.pn infection
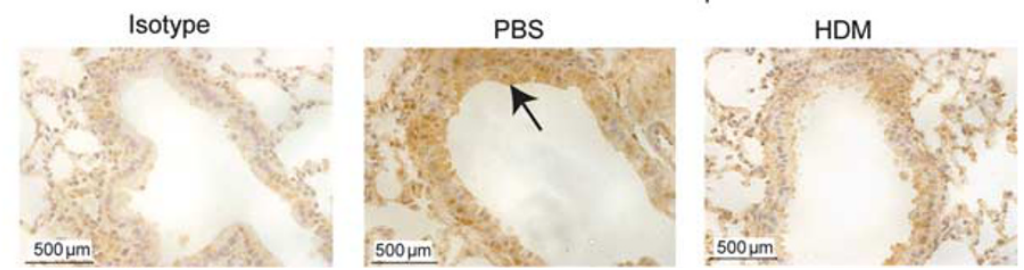

Figure 2 Reduced neutrophil numbers and production of proinflammatory cytokines in house-dust mite (HDM)-exposed mice following bacterial challenge. (a) Airway and (b) lung neutrophil $\left(\mathrm{CD} 11 \mathrm{~b}^{+} \mathrm{Ly}_{6 \mathrm{G}}{ }^{+}\right)$recruitment in HDM- or phosphate-buffered saline (PBS)-exposed mice were compared after intranasal administration of Streptococcus pneumoniae (S.pn). Data are presented as box and whisker plots with the median. ${ }^{\star} P<0.05$;

${ }^{\star \star} P<0.01$. Data show representative samples of five mice per group from one experiment and are representative of three independent experiments. Quantification of (c) tumor necrosis factor (TNF), (d) interleukin (IL)-6, (e) CXCL1 (KC), (f) macrophage inflammatory protein (MIP)-2 and (g) matrix metalloproteinase (MMP)-9 in the bronchoalveolar lavage fluid by enzyme-linked immunosorbent assay. Data presented are the means $\pm \mathrm{s}$.d. ${ }^{*} P<0.05$; ${ }^{\star \star} P<0.01 ;{ }^{* \star *} P<0.001$. (h) Immunohistology of formalin-fixed lung tissue for KC. Brown colored stain (arrows) shows KC on the lung sections detected by HRP-DAB and examined by light microscopy (original magnification $\times 40$ ) and representative of the lungs from five mice per group. NS, not significant.

with PBS, exposure. This coupled with reduced TLR2 expression may prevent neutrophil recruitment/activation in the HDM-sensitized airway. TREM-2 has, in the past, been attributed a role in osteoclastogenesis and is thought to inhibit TLR responses. However, recent studies imply that it is also a phagocytic receptor for bacteria. ${ }^{16} \mathrm{~A}$ transient increase in TREM-2-expressing airway macrophages was observed in HDM-sensitized mice $78 \mathrm{~h}$ following HDM cessation (Figure 3g,h). However, if TREM-2 is a bacterial receptor in this setting, the increase is clearly not enough to overcome the 
defect in other antibacterial defences. The reduced inflammatory cytokines and TLR expression may be due to the upregulation of endogenous negative regulatory pathways. Reverse transcriptase-PCR analysis of whole-lung homogenates shows that the TLR signaling inhibitors A20 (that restricts TLR responses by regulating the ubiquitination of key signaling proteins such as Traf6 ${ }^{17}$ ), IRAK-M (that binds to MyD88 and TRAF6, blocking TLR action by stabilizing the signaling complex ${ }^{18}$ ), and Tollip (that interacts with IL-1R, TLR2, and TLR4 to prevent cell activation and inhibits IRAK autophosphorylation $^{19}$ ) are all increased in HDM- compared with PBS-sensitized mice. Furthermore, TRIAD3A (an E3 ubiquitin-protein ligase that enhances ubiquitination and proteolytic degradation of some TLRs) was also increased (Figure 3i), though it has not yet been shown to act on TLR2. ${ }^{20}$ Inhibition of TLR responsiveness can also occur by the upregulation of receptors that recruit similar adapter molecules to initiate signaling. In this respect it is interesting that the IL-33 receptor, ST2L (associated with development of allergic airway disease), that recruits the TLR-signaling adapter MyD88 ${ }^{10}$ is raised on CD11c + airway macrophages (Figure $3 \mathbf{j}$ ) together with the negative regulator CD200R (Figure 3k) in the HDM-sensitized lung at the onset of bacterial infection.

\section{Instillation of KC/MIP-2 to the HDM-exposed lung restores antibacterial immunity}

The HDM-exposed lung is clearly deficient in multiple factors required for clearance of $S$. pneumoniae, and this deficit likely occurs at the level of innate recognition and responsiveness of TLRs. We therefore attempted to bypass this deficiency by restoring the neutrophil chemoattractants, KC or MIP-2. Intranasal administration of KC $72 \mathrm{~h}$ following cessation of HDM (Figure 4a) restored $\mathrm{Ly}_{6 \mathrm{G}}{ }^{+}$neutrophil numbers in the airway (Figure $4 \mathbf{b}$ ) and lung (Figure 4c). This effect persisted also through bacterial challenge, where neutrophil numbers even exceeded those in the PBS control group upon administration of KC (Figure 4f) or MIP-2 (Figure 4g). Bacterial colony-forming units in the airway and lung were significantly reduced in HDMsensitized mice in which KC (Figure 4d) or MIP-2 (Figure 4e) had been restored.

\section{Exacerbation of bacterial infection wanes without re-exposure to allergen}

In order to determine whether the timing of bacterial exposure is critical, mice were exposed to HDM on alternate days for 3 weeks and 3,7,10, or 14 days after the last HDM exposure infected with S. pneumoniae (Figure 5a). Invasion of lung tissue with bacteria was lost after an absence of HDM for 7 days. The proportion of mice harboring bacteria at $48 \mathrm{~h}$ after $S$. pneumoniae infection also reduced after day 3 , but never reached the condition experienced by PBS-exposed mice (Figure 5b). The improvement with increasing time post allergen exposure was associated with a return in the ability to recruit neutrophils to the airspaces (Figure 5c) and a decrease in eosinophils (Figure 5d). This suggests that allergen exposure causes increased susceptibility to invasive bacterial infection within a specific time window, and that allergen load may be a factor in determining the seriousness of concomitant bacterial infection.

\section{DISCUSSION}

Our results show that the allergen-exposed lung is defective in its ability to respond to bacteria, due to the upregulation of endogenous TLR-negative regulators and pathways that compete for intracellular adapters (e.g., sequestration of MyD88 by the IL-33 receptor, ST2L) and an imbalance in pathways designed to support (TREM-1) or inhibit (TREM2, CD200R) TLR signaling. This culminates in an inability to recruit neutrophils and ultimately results in bacterial invasion and bacteremia. Obviously we are presenting the worse-case scenario as we instil a bacterial innoculum that causes a self-limiting infection in $B A L B / c$ mice (in C57BL/6 mice lower innocula produce a similar effect). In patients, bacteria may be encountered in the environment or from the commensal flora at lower concentrations. Our data do not represent a simple imbalance of Th1/Th17 vs. Th2 cytokines, but a specific alteration at the level of innate immunity to the bacteria. Indeed, we were surprised by the lack of involvement of IL-17A in our study, as this cytokine is involved in neutrophil recruitment in another lung allergic/infection model. This latter study, however, examines $H$. influenzae administered during ovalbumin peritoneal sensitization, but before allergen challenge, and does not examine the effect of allergy on bacterial clearance. ${ }^{21}$ The novel alterations in antibacterial immunity that we identify are all amenable to therapeutic manipulation, which may reduce the symptoms of allergy in addition to restoring the ability to respond to bacteria. Despite the variety of alterations in innate immunity, KC or MIP-2 administered directly to the allergen-exposed lung reverses bacterial susceptibility, and neutrophil recruitment is restored. This suggests that the most important influence of HDM with regard to subsequent bacterial infection is at the level of cells required to produce neutrophil chemoattractants (most likely airway epithelium and/or luminal macrophages) and is supported by an early reduction

Figure 3 Reduced expression of pathogen-recognition receptors on macrophages and neutrophils in allergic mice compared with phosphatebuffered saline (PBS) controls. Expression of surface Toll-like receptor (TLR)-2 involved in bacterial recognition on (a, b) airway neutrophils and $(\mathbf{c}, \mathbf{d}) \mathrm{CD} 11 \mathrm{c}^{+}$airway macrophages is shown as percentage of cells and histograms. Percentage and total cell count of $(\mathbf{e}, \mathbf{f})$ triggering receptor expressed on myeloid cells (TREM)1-positive neutrophils in the airways and $(\mathbf{g}, \mathbf{h})$ TREM2-positive alveolar macrophages $\left(\mathrm{CD} 11 \mathrm{c}^{+}\right) 78 \mathrm{~h}$ after final house-dust mite (HDM) exposure. (i) Expression of TLR-negative regulators in whole lung of allergic mice was compared with PBS controls. The level of different RNA transcripts, as indicated, was assessed by real-time PCR. All values were normalized against the expression of $18 \mathrm{~S}$ and then to a control (PBS) sample. Percent of CD11c + macrophages expressing (j) ST2L or (k) CD200R was determined by flow cytometry. Data presented as box and whisker plots with the median of $n=5$ mice in two independent experiments. ${ }^{*} P<0.05 ;{ }^{* *} P<0.01$. S.pn, Streptococcus pneumoniae. 
in MIP-2 and MMP9 followed by a later effect on neutrophil numbers. This desensitization of innate immunity in the allergic lung is remarkably similar to bacterial susceptibility following influenza infection, where again TLR-negative regulators are enhanced, leading to reduced neutrophilia and bacterial dissemination, ${ }^{22-24}$ though our current study extends further into novel TLR regulators. The reprogramming of lung innate immunity therefore persists for prolonged periods of time and
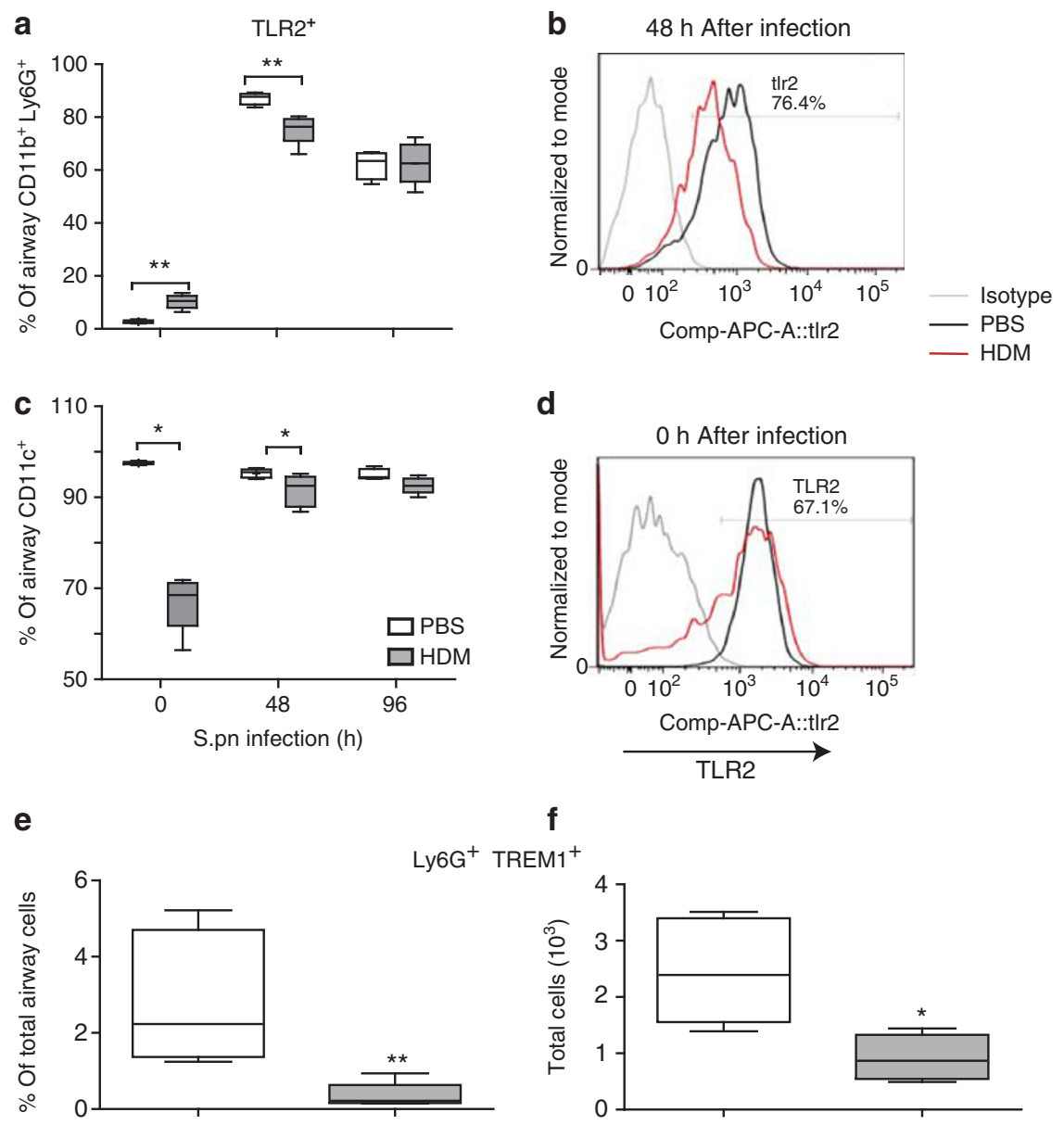

f

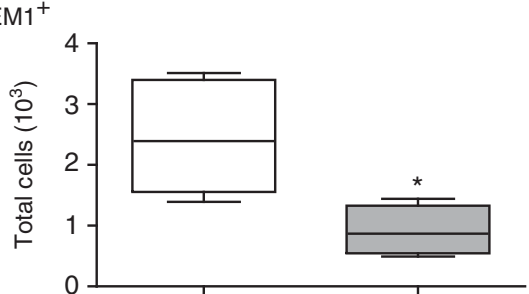

g

h

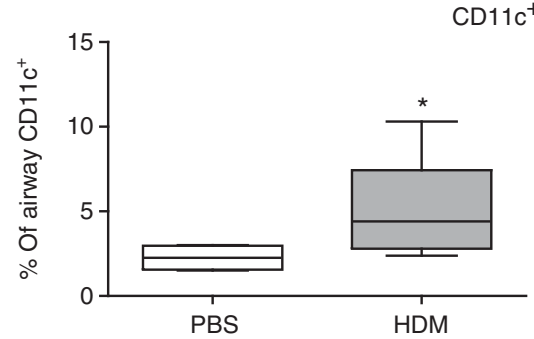

EMM $2^{+}$

i
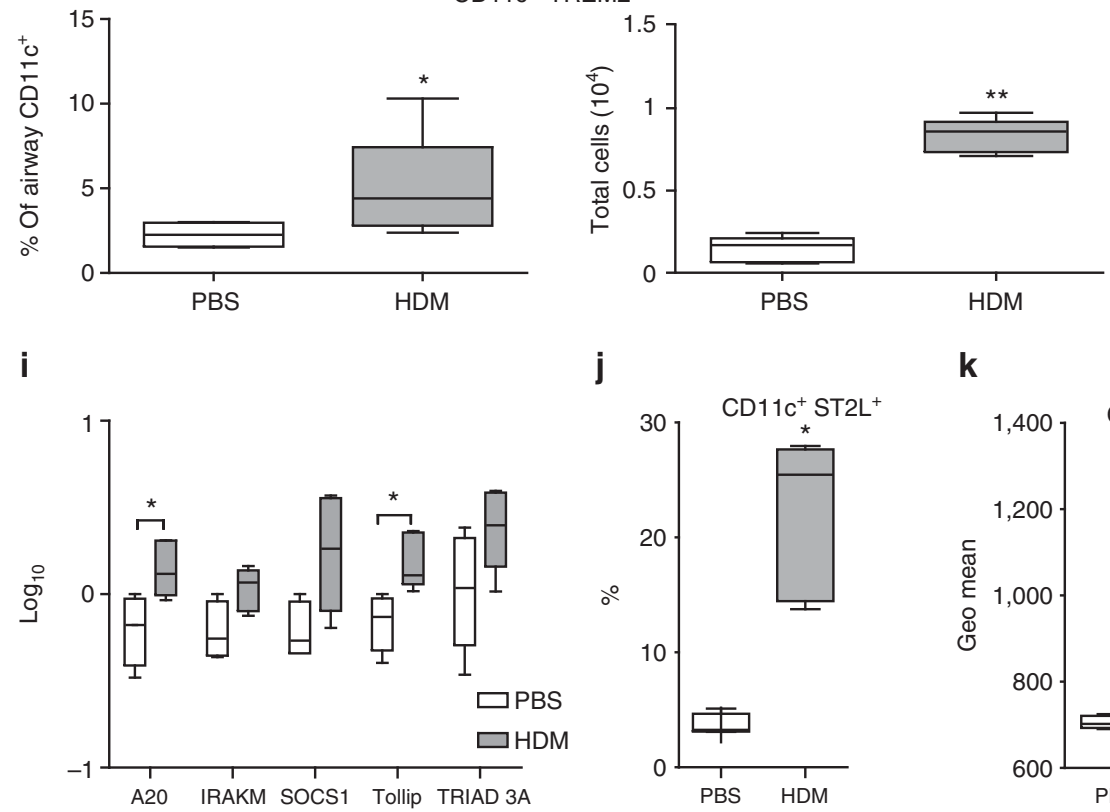

j

k

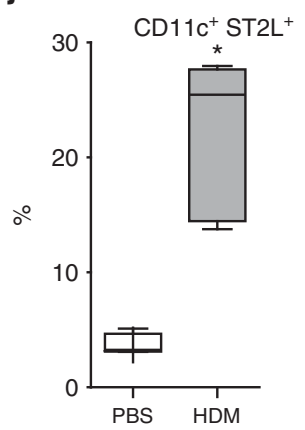


a

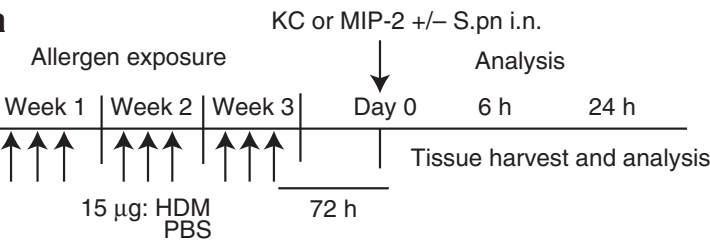

b

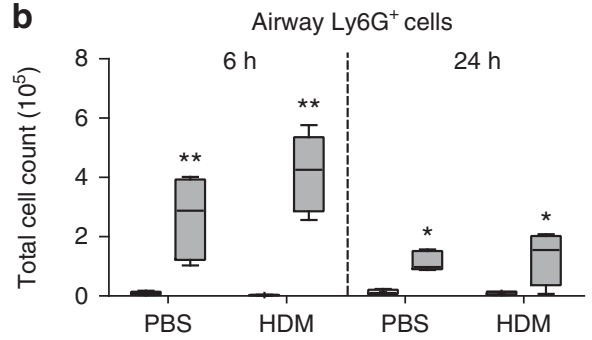

d

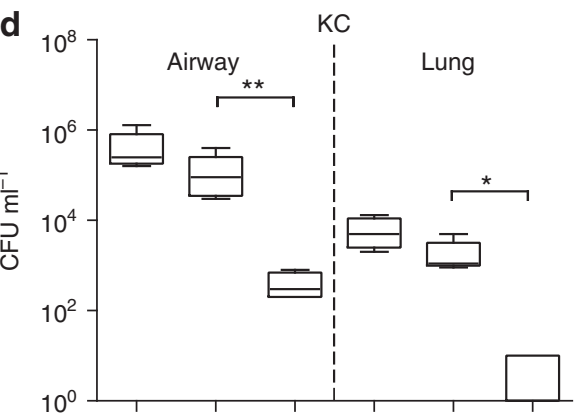

f

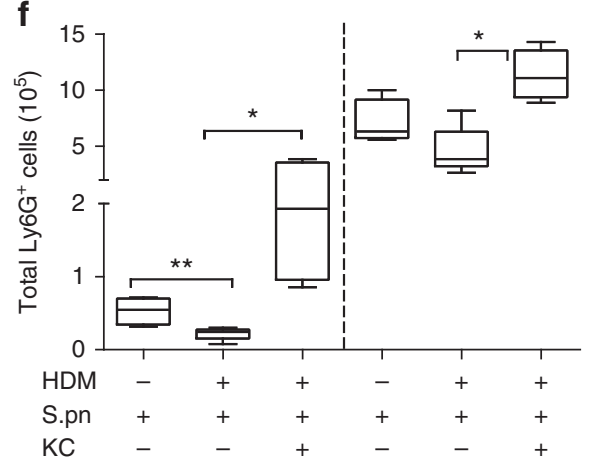

C

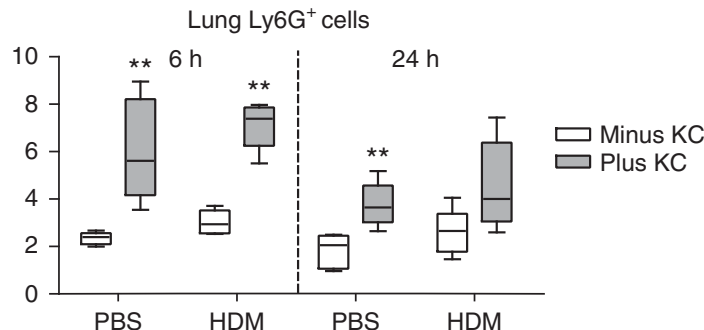

e
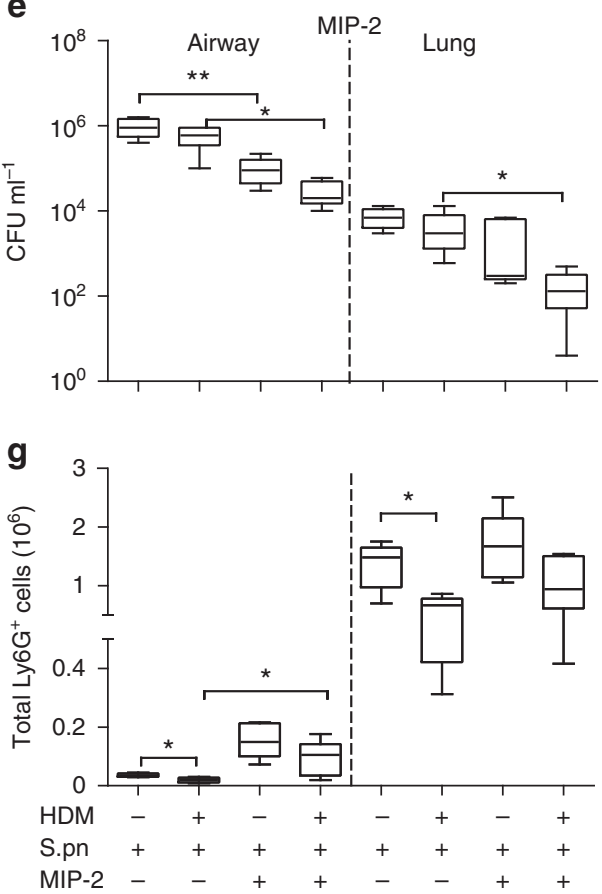

Figure 4 Equivalent numbers of neutrophils recruited into the airways and lungs of control (phosphate-buffered saline (PBS)) and house-dust mite (HDM)-exposed mice after recombinant CXCL1 (KC) or macrophage inflammatory protein (MIP)-2 instillation. (a) An illustration of KC or MIP-2 with or without S. pneumonia (S.pn) administration protocol. (b) Airway and (c) lung neutrophil $\left(\mathrm{Ly}_{6 \mathrm{G}}{ }^{+}\right.$) recruitment in HDM- or PBS-exposed mice were analyzed 6 and $24 \mathrm{~h}$ after intranasal (i.n.) administration of $1 \mu \mathrm{g}$ of recombinant murine $\mathrm{KC}$ per mouse on day 0 . Airway and lung bacterial titres were determined $6 \mathrm{~h}$ after $(\mathbf{d})$ infection $\pm \mathrm{KC}$ or $(\mathbf{e})$ MIP-2. (f, $\mathbf{g})$ Administration of $1 \mu \mathrm{g}$ recombinant murine KC (f) or MIP-2 (g) per mouse overcomes the inability of HDM-exposed mice to recruit neutrophils $6 \mathrm{~h}$ after bacterial infection. Box and whisker plots of five mice per group representative of two experiments. ${ }^{*} P<0.05 ;{ }^{* \star} P<0.01$. CFU, colony-forming units.

follows significant inflammation, whether driven by an allergen or a virus. We do not suggest that this occurs in all inflammatory situations, but occurs only in those most severely affected. Though desensitization and bacterial complications reduce as the time to last allergen exposure increases, susceptibility above non-allergic mice remains.

Many studies imply that bacterial infection causes exacerbation of more chronic Th2-driven asthma in adults ${ }^{25-27}$ and acute wheezy episodes in children. ${ }^{28-30}$ Sequencing of bacterial $16 \mathrm{~s}$ ribosomal RNA in patients with asthma shows that Prevotella, Neiserria, Streptococcus, Moraxella, and Haemophilus species dominate. ${ }^{31}$ However, the response of patients with established Th2-driven asthma to antibiotics such as the ketolide antibiotictelithromycin suggests that acute and chronic bacterial infections may have a significant role in asthma exacerbation. ${ }^{32}$ We now show, in a more transient Th2 model of HDM-induced airway inflammation, that the underlying condition may actually cause an exacerbated bacterial infection, ${ }^{33,34}$ similar to 
a

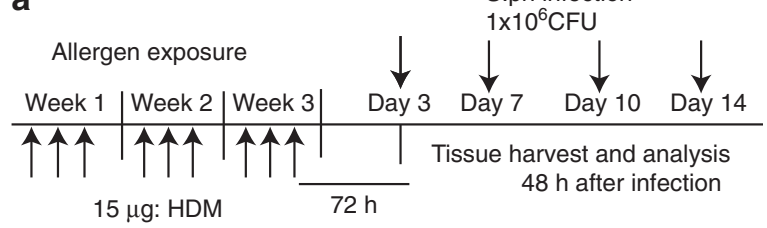

b
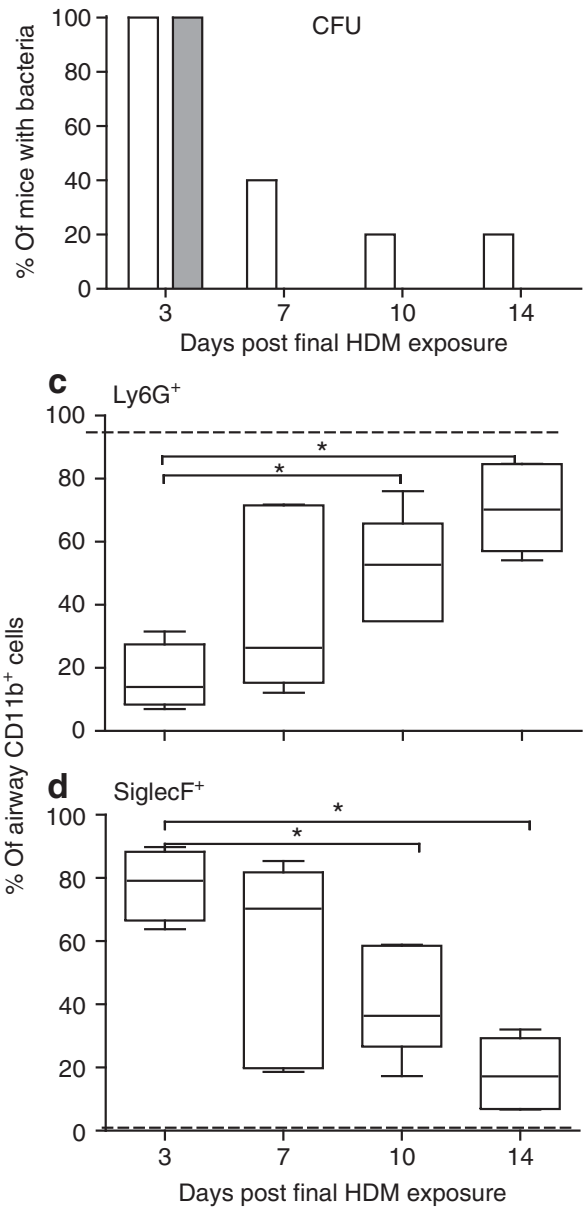

Figure 5 The percentage of mice with bacteria reduces and the numbers of neutrophils in the airways of house-dust mite (HDM)/ Streptococcus pneumoniae (S.pn)-challenged mice increases as HDM exposure wanes. (a) Experimental design: five BALB/c mice per group were inoculated intranasally with $15 \mu \mathrm{g}$ of HDM three times a week for 3 weeks and subsequently infected on days $3,7,10$, and 14 after final HDM administration and harvested $48 \mathrm{~h}$ after infection. (b) The percentage of mice with bacteria reduces as HDM exposure wanes. (c) The frequency of $C D 11 b^{+}{ }^{2} 6 G^{+}{ }^{+}$neutrophils and (d) $C D 11 b^{+}$ SiglecF $\mathrm{F}^{+}$in the airways was determined by flow cytometry. The dotted line illustrates phosphate-buffered saline/S.pn-infected mice harvested $48 \mathrm{~h}$ after infection (baseline). Data are presented as box and whisker plots, with the median of $n=5$ mice per group in two independent experiments. ${ }^{\star} P<0.05$. BAL, bronchoalveolar lavage; CFU, colonyforming units.

the observed downregulation of TLR2 and IL- 6 and delayed clearance of Mycoplasma pneumoniae in ovalbumin-exposed mice. Furthermore, SPLUNC1 (short palate, lung, and nasal epithelium clone 1) protein is produced by epithelial cells and is thought to possess antibacterial properties, but is reduced in mouse models of allergy, ${ }^{35}$ though we did not observe this reduction in HDM-induced airway inflammation. Asthma is a chronic lung condition characterized by persistent Th2driven eosinophilia with a greater than twofold increased risk of invasive pneumococcal disease, and with an estimated population-attributable risk of $11 \%$ where S. pneumoniae has been described. ${ }^{3}$ Furthermore, a retrospective study found an attributable bacterial risk of $17 \% .^{36}$ Therefore, underlying Th2 conditions, whether transient or chronic, may impede antibacterial immunity. However, it should also be noted that bacterial colonization is observed before the development of Th2-associated airway disease. Asymptomatic neonates whose throats are colonized with S. pneumoniae, H. influenzae, or M. catarrhalis are at increased risk for recurrent wheeze and asthma early in life. ${ }^{37}$ The role of bacteria in the initiation of allergic airway disease and in its exacerbation are likely to be very different; for example, an influence of bacterial proteases in the former and bacterial outgrowth in the latter.

In our study, we focus on TLR2 responsiveness in the allergen-exposed lung as bacterial LTA is the primary PAMP and is recognized by it. We cannot, however, rule out an effect on other TLRs and related molecules. Both Der p1 and S. pneumoniae mediate TLR4 signaling that is regulated by a number of the inhibitors we measured. The phenomenon of TLR cross-tolerance is a process whereby TLR-activated cells are, for a short period of time, refractory to subsequent TLR stimulation. ${ }^{38}$ Der $\mathrm{p} 2$ and Der $\mathrm{p} 7$ have structural and functional homology with MD-2; the lipopolysaccharide-binding component of the TLR4 signaling complex ${ }^{39,40}$ and HDM extracts are known to contain low levels of lipopolysaccharideand so it is possible that we may be looking at a similar refractory effect via TLRs alone. Cross-tolerance, however, is only reported to last for a few hours, whereas bacterial invasiveness of the allergen-exposed lung lasts for days and their persistence in the airspaces for weeks.

Neutrophils are clearly important in lung antibacterial immunity as their absence leads to lethal S. pneumoniae infection. ${ }^{41}$ A reduced number of neutrophils may compound the problem, as their production of MMP9 is required to enhance the chemotactic activity of CXCL8 (IL-8) via cleavage ${ }^{42}$ and MMP9deficient mice succumb to abdominal sepsis. ${ }^{43}$ It is, therefore, interesting that we observed decreased MMP9 in mice with HDM-induced allergic airway disease. Furthermore, neutrophils secrete IFN- $\gamma$ in the early response to $S$. pneumoniae infection in mice $^{44}$ and thus may assist the development of appropriate T-cell and antibody subtypes, in addition to their release of reactive oxygen species, antimicrobial proteins, and neutrophil extracellular traps. ${ }^{45-47}$ The data presented here show that allergenexposed mice have a fundamental inability to mount effective bacterial clearance. The discovery of the molecular mechanism behind this defect will enable development of specific therapy to limit the physiological consequences.

\section{METHODS}

Animals. Female BALB/c mice (Harlan Olac, Bicester, UK) weighing 17-19g were maintained in specific pathogen-free conditions at Bio Safety Level 2. Mice were exposed to HDM extract i.n. (Greer Laboratories, 
Lenoir, NC; Dermatophagoides pteronyssinus (Der p1) $149.02 \mu \mathrm{g}$ per vial, endotoxin $125 \mathrm{EU}$ per vial, $4.29 \mathrm{mg}$ protein per vial and $11.90 \mathrm{mg}$ dry weight per vial). In all, $15 \mu \mathrm{g}$ of protein in $15 \mu \mathrm{l}$ of PBS or $15 \mu \mathrm{l}$ PBS alone (for controls) was administered i.n. on 3 alternate days a week for 3 weeks. Seventy-two hours (or at later time points shown in the text) following the last HDM exposure, $1 \times 10^{6} \mathrm{CFU}$ (colony-forming units) of S. pneumoniae strain D39 (NCTC7466, National Collection of Type Cultures, UK) was administered i.n. In some experiments, $1 \mu \mathrm{g}$ of recombinant murine KC or MIP-2 (Peprotech EC, Ltd, UK; KC Cat. No: 250-11 and MIP-2 Cat. No: 250-15) was given i.n. either alone (in $20 \mu \mathrm{l}$ of sterile PBS) or with $S$. pneumoniae at the time of bacterial challenge (in $50 \mu \mathrm{l}$ of sterile PBS). At various time points following bacterial infection, mice were administered $3 \mathrm{mg}$ pentobarbitone and exsanguinated via the femoral artery. All animal procedures and care conformed strictly to the United Kingdom Home Office Guidelines under the Animals (Scientific Procedures) Act 1986 and the protocols were approved by the Home Office of Great Britain (License number: 70/6646).

Streptococcus pneumoniae (D39). Bacteria were cultured at $37^{\circ} \mathrm{C}$ in $5 \% \mathrm{CO}_{2}$ in Todd-Hewitt broth (Sigma, Dorset, UK) supplemented with $0.5 \%$ yeast extract (OXOID) to an OD 600 of 0.4 (approximately $2 \times 10^{8}$ $\mathrm{CFU} \mathrm{ml} l^{-1}$ ) and stored at $-80^{\circ} \mathrm{C}$ in $10 \%$ glycerol as single-use aliquots. The bacterial titer from the airway, lung, and blood at the time points specified was determined by counting colonies from serial tenfold dilutions of single-cell suspensions that had been plated on Columbia blood agar supplemented with 5\% defibrinated horse blood. The total number of colony-forming units per tissue was determined as the number of colonies $\times$ the dilution factor $\times$ the original cell suspension volume.

Cell recovery and isolation. Bronchoalveolar lavage, lung tissue, and serum were harvested as described previously. ${ }^{22}$ Briefly, blood clotting was prevented using $100 \mathrm{U} \mathrm{ml}^{-1}$ heparin. The lungs were inflated three times with $1.5 \mathrm{ml} 1 \mathrm{mM}$ ethylenediaminetetraacetic acid in Hank's buffered salt solution via an intratracheal cannula, centrifuged for $5 \mathrm{~min}(\mathrm{~min})$ at $240 \times \mathrm{g}$, and the supernatant stored at $-80^{\circ} \mathrm{C}$. Lung tissue was finely minced and digested for $30 \mathrm{~min}$ at $37^{\circ} \mathrm{C}$, while agitated with Liberase (Roche, West Sussex, UK, 0.4 Wunsch units $\mathrm{ml}^{-1}$ ) and DNase (Roche, $50 \mu \mathrm{g} \mathrm{ml}^{-1}$ ). This digested preparation was disrupted to a single-cell suspension by passage through a $100-\mu \mathrm{m}$ sieve (BD Labware, Franklin Lake, NJ), spun for $5 \mathrm{~min}$ at $240 \times g$, and red blood cells lysed by resuspending pellets in $0.15 \mathrm{M}$ ammonium chloride, $1 \mathrm{M}$ potassium hydrogen carbonate, and $0.01 \mathrm{mM}$ ethylenediaminetetraacetic acid, $\mathrm{pH} 7.2$ for $3 \mathrm{~min}$ at room temperature before spinning $(240 \times g$ for $5 \mathrm{~min})$ and washing with RPMI with $10 \%$ fetal calf serum (R10F). Airway and lung cell viability was assessed by trypan blue exclusion and cells resuspended in R10F at $1 \times 10^{6}$ cells ml $^{-1}$. In some experiments, a lung lobe was fixed with $10 \%$ neutral-buffered formalin in PBS and embedded in paraffin wax and subsequently $4-\mu \mathrm{m}$ sections were stained as described below.

Flow cytometry. Single-cell suspensions were stained for surface markers and intracellular cytokines as indicated in the text in PBS containing $0.1 \%$ sodium azide and $1 \%$ bovine serum albumin (PBA) for $30 \mathrm{~min}$ at $4{ }^{\circ} \mathrm{C}$ and fixed with $2 \%$ paraformaldehyde. Neutrophils were identified as Ly6g + , F4/80-, CD11c-, CD11b +, Siglec F-. To detect intracellular cytokines, $10^{6} \mathrm{cells} \mathrm{ml}^{-1}$ were incubated with $50 \mathrm{ng} \mathrm{ml}^{-1} \mathrm{PMA}$, $500 \mathrm{ng} \mathrm{ml}^{-1}$ ionomycin (Calbiochem, Nottingham, UK), and $10 \mu \mathrm{g} \mathrm{ml}^{-1}$ brefeldin $\mathrm{A}$ for $3 \mathrm{~h}$ at $37^{\circ} \mathrm{C}$. Cells were then stained with anti-CD4 and anti-CD8 and fixed as described above. After permeabilization with PBS containing $1 \%$ saponin/ $1 \%$ bovine serum albumin $/ 0.05 \%$ azide (saponin buffer) for $10 \mathrm{~min}$, cells were stained with anti-IL-17A (eBioscience, Hatfield, UK) diluted 1:50 in saponin buffer. Thirty minutes later, cells were washed once in saponin buffer and once in PBA. All antibodies were purchased from BD Pharmingen (Oxford, UK), R\&D Systems (Abingdon, UK), or eBioscience, UK. Data acquired on a BD FACS Canto II and 30,000 lymphocyte or myeloid events analyzed with the FlowJo (Treestar, Ashland, OR) analysis programme.
Forward scatter and side scatter gates were used to exclude debris in organ homogenates. In some experiments, dead cells were excluded using a fixable near-IR dead cell stain kit for 633 or 635-nm excitation (Invitrogen, Paisley, UK; L10119).

Histological analysis. Paraffin-embedded sections were stained with hematoxylin/eosin to evaluate general morphology. Goblet cells were visualized on periodic acid-Schiff-stained lung sections and scored as previously described. ${ }^{48}$ Hematoxylin/eosin and PAS staining were done by Lorraine Lawrence, Imperial College London. Image analysis was performed using Scion Image (Scion, Frederick, MD).$^{49}$ For KC analysis, papaffin-embedded lung sections were incubated with goat anti-mouse KC IgG $\left(15 \mu \mathrm{g} \mathrm{ml}^{-1}\right.$, R\&D systems Cat: AF-453-NA) and detected with anti-goat HRP-DAB cell staining kit (R\&D systems; CTS008). Sections were counter-stained with hematoxylin, mounted in DPX, and photographed using a light microscope.

Cytokine enzyme-linked immunosorbent assay. Tumor necrosis factor, KC, MIP-2, MMP-9 (all R\&D systems), and IL-6 and IL-17A (eBioscience) in bronchoalveolar lavage fluid were quantified using Duoset ELISA kits following the manufacturer's instructions. Microtiter plates (Nunc, Roskilde, Denmark) were coated with $100 \mu$ l of capture antibody diluted in the recommended buffer and incubated according to the manufacturer's protocol. After 3-5 washes with PBS containing $0.5 \%$ Tween 20, plates were blocked with $200 \mu$ lof the recommended diluent and left for $1 \mathrm{~h}$ at room temperature. Samples and standards (diluted in reagent diluent) were then incubated for a further $2 \mathrm{~h}$ at room temperature. After 3-5 washes, bound cytokine was detected using biotinylated antibodies with avidin-horseradish peroxidase followed by tetramethylbenzidine. Optical densities were read at $450 \mathrm{~nm}$ using $570 \mathrm{~nm}$ as a reference. The mean optical density of blank wells (no cytokine) was subtracted from the results obtained for samples and standards. A standard curve was used to calculate the concentration of cytokine in each sample.

Isolation of mRNA and real-time PCR. Total lung RNA was extracted using RNeasy Mini kit (QAIGEN, West Sussex, UK), reverse transcribed into cDNA (SuperScript III Reverse transcriptase, Invitrogen), and then amplified using specific TaqMan primers and probes for the TLR-negative regulators tested. All values were normalized against the expression of $18 \mathrm{~S}$ and then to a control (PBS sensitized) sample using ABI 7900HT (Applied Biosystems, CA) sequence detection system and software. The primers for the genes of interest were purchased from Applied Biosystems: Tollip (Mm00445841), IRAKM (Mm00518541), A20 (Mm00437121) SOCS1 (Mm00782550), and TRIAD3A (Mm01205634).

Analysis of bone marrow neutrophils. Bone marrow from mouse femurs was flushed with Hank's buffered salt solution containing hydroxyethyl piperazineethanesulfonic acid $30 \mathrm{mM}$ and ethylenediaminetetraacetic acid $15 \mathrm{mM}$ using a $23-\mathrm{G}$ needle. Clumps were disrupted using a 21-G needle and the suspension was then centrifuged at 1,200 r.p.m. for $5 \mathrm{~min}$. Red blood cells were lysed using $3 \mathrm{ml} 0.2 \% \mathrm{NaCl}$ followed immediately by $3 \mathrm{ml} 1.6 \% \mathrm{NaCl}$ and made up to $20 \mathrm{ml}$ with RPMI and $0.1 \%$ bovine serum albumin. Cell viability was assessed using trypan blue exclusion and subsequently re-suspended in RPMI with $0.1 \%$ bovine serum albumin. Cells were stained for neutrophils as described above.

Analysis of airway antimicrobial substances. Airway C-reactive protein was quantified by enzyme-linked immunosorbent assay according to the manufacturer's instructions (R\&D systems). SPLUNC1 was detected by western blotting. Briefly, all samples were electrophoresed by reducing sodium dodecyl sulfate-polyacrylamide gels and electroblotted onto nitrocellulose membranes. Membranes were blocked in PBS containing $5 \%$ milk and $0.1 \%$ Tween- 20 for $2 \mathrm{~h}$ at room temperature and incubated with sheep anti-mouse PLUNC (SPLUNC1; $1 \mu \mathrm{g} \mathrm{ml}^{-1}$, R\&D systems) diluted in $5 \%$ milk/PBS $/ 0.1 \%$ Tween-20, overnight at $4^{\circ} \mathrm{C}$. After three washes of $15 \mathrm{~min}$ in PBS/0.1\% Tween-20, membranes were incubated 
with Donkey anti-sheep IgG-HRP (1:1,000 dilution; R\&D systems) and detected using ECL chemiluminescent kits (Pierce, Rockford, IL).

Statistics. GraphPad Prism 5 software was used for all statistical calculations. For comparison with PBS controls, the statistical test used was Mann-Whitney. Data are presented as box and whisker plots, with the box showing the median and the 25 th and 75 th percentiles. Whiskers of the graph show the largest and smallest values. $P$ values $<0.05$ were considered significant.

SUPPLEMENTARY MATERIAL is linked to the online version of the paper at http://www.nature.com/mi

\section{ACKNOWLEDGMENTS}

We thank Natasha Strydom and Robert Snelgrove for advice on bone marrow isolation and western blotting, respectively, and Lorraine Lawrence for histological sectioning. TH, JG, and JIS were supported by MRC (Refs: G0802752 and P171/03/C1/048), MH by MRC-Asthma UK Centre in Allergic Mechanisms of Asthma, and CML by the Wellcome Trust (Ref 057704).

\section{AUTHOR CONTRIBUTION}

$\mathrm{TH}$, JIS and CML made substantial contributions to the conception and design of this work and interpretation of data. MH, JIS, and JG acquired, analyzed, and interpreted the data. All authors assisted in drafting the article and revising it critically. All authors approved the final version to be published.

\section{DISCLOSURE}

The authors declared no conflict of interest.

(C) 2012 Society for Mucosal Immunology

\section{REFERENCES}

1. Mulholland, K. Childhood pneumonia mortality--a permanent global emergency. Lancet 370, 285-289 (2007).

2. Jounio, U. et al. Pneumococcal carriage is more common in asthmatic than in non-asthmatic young men. Clin. Respir. J. 4, 222-229 (2010).

3. Talbot, T.R. et al. Asthma as a risk factor for invasive pneumococcal disease. N. Engl. J. Med. 352, 2082-2090 (2005).

4. Kraft, M. The role of bacterial infections in asthma. Clin. Chest Med. 21, 301-313 (2000).

5. Blasi, F. \& Johnston, S.L. The role of antibiotics in asthma. Int. J. Antimicrob. Agents 29, 485-493 (2007).

6. Paterson, G.K. \& Orihuela, C.J. Pneumococci: immunology of the innate host response. Respirology 15, 1057-1063 (2010).

7. Pittet, L.A. et al. Earliest innate immune responses require macrophage RelA during pneumococcal pneumonia. Am. J. Respir. Cell Mol. Biol. 45, 573-581 (2011).

8. Malley, R. Antibody and cell-mediated immunity to Streptococcus pneumoniae: implications for vaccine development. J. Mol. Med. 88, 135-142 (2010).

9. Kolls, J.K. Th17 cells in mucosal immunity and tissue inflammation. Semin. Immunopathol. 32, 1-2 (2010).

10. Lloyd, C.M. IL-33 family members and asthma - bridging innate and adaptive immune responses. Curr. Opin. Immunol. 22, 800-806 (2010).

11. Gregory, L.G. \& Lloyd, C.M. Orchestrating house dust mite-associated allergy in the lung. Trends Immunol. 32, 402-411 (2011).

12. Gregory, L.G. et al. Overexpression of Smad2 drives house dust mitemediated airway remodeling and airway hyperresponsiveness via activin and IL-25. Am. J. Respir. Crit. Care Med. 182, 143-154 (2010).

13. Kumar, V. \& Sharma, A. Neutrophils: cinderella of innate immune system. Int. Immunopharmacol. 10, 1325-1334 (2010).

14. Gregory, L.G. et al. Inhaled house dust mite induces pulmonary T helper 2 cytokine production. Clin. Exp. Allergy 39, 1597-1610 (2009).

15. Klesney-Tait, J., Turnbull, I.R. \& Colonna, M. The TREM receptor family and signal integration. Nat. Immunol. 7, 1266-1273 (2006).

16. Qiu, H., Kuolee, R., Harris, G. \& Chen, W. Role of NADPH phagocyte oxidase in host defense against acute respiratory Acinetobacter baumannii infection in mice. Infect. Immun. 77, 1015-1021 (2009).
17. O'Neill, L.A. When signaling pathways collide: positive and negative regulation of toll-like receptor signal transduction. Immunity $\mathbf{2 9}, 12-20$ (2008).

18. Kobayashi, K. et al. IRAK-M is a negative regulator of Toll-like receptor signaling. Cell 110, 191-202 (2002).

19. Zhang, G. \& Ghosh, S. Negative regulation of toll-like receptor-mediated signaling by Tollip. J. Biol. Chem. 277, 7059-7065 (2002).

20. Chuang, T.H. \& Ulevitch, R.J. Triad3A, an E3 ubiquitin-protein ligase regulating Toll-like receptors. Nat. Immunol. 5, 495-502 (2004).

21. Essilfie, A.T. et al. Haemophilus influenzae infection drives IL-17-mediated neutrophilic allergic airways disease. PLoS Pathog. 7, e1002244 (2011).

22. Goulding, J. et al. Lowering the threshold of lung innate immune cell activation alters susceptibility to secondary bacterial superinfection. J. Infect. Dis. 204, 1086-1094 (2011).

23. Snelgrove, R.J. et al. A critical function for CD200 in lung immune homeostasis and the severity of influenza infection. Nat. Immunol. 9, 1074-1083 (2008).

24. Didierlaurent, A. et al. Sustained desensitization to bacterial Toll-like receptor ligands after resolution of respiratory influenza infection. J. Exp. Med. 205, 323-329 (2008).

25. Kraft, M. et al. Detection of Mycoplasma pneumoniae in the airways of adults with chronic asthma. Am. J. Respir. Crit. Care Med. 158, 998-1001 (1998).

26. Leaver, R. \& Weinberg, E.G. Is Mycoplasma pneumoniae a precipitating factor in acute severe asthma in children? S. Afr. Med. J. 68, 78-79 (1985).

27. Pasternack, R., Huhtala, H. \& Karjalainen, J. Chlamydophila (Chlamydia) pneumoniae serology and asthma in adults: a longitudinal analysis. J. Allergy Clin. Immunol. 116, 1123-1128 (2005).

28. Bisgaard, $\mathrm{H}$. et al. Association of bacteria and viruses with wheezy episodes in young children: prospective birth cohort study. Br. Med. J. 341, c4978 (2010)

29. Watson, K. et al. Upper respiratory tract bacterial carriage in Aborigina and non-Aboriginal children in a semi-arid area of Western Australia. Pediatr. Infect. Dis. J. 25, 782-790 (2006).

30. Harrison, L.M., Morris, J.A., Telford, D.R., Brown, S.M. \& Jones, K. The nasopharyngeal bacterial flora in infancy: effects of age, gender, season, viral upper respiratory tract infection and sleeping position. FEMS Immunol. Med. Microbiol. 25, 19-28 (1999).

31. Hilty, M. et al. Disordered microbial communities in asthmatic airways. PLOS ONE 5, e8578 (2010).

32. Johnston, S.L. et al. The effect of telithromycin in acute exacerbations of asthma. N. Engl. J. Med. 354, 1589-1600 (2006).

33. Hartert, T.V. Are persons with asthma at increased risk of pneumococcal infections, and can we prevent them? J. Allergy Clin. Immunol. 122, 724-725 (2008).

34. Sevin, C.M. \& Peebles, R.S. Jr. Infections and asthma: new insights into old ideas. Clin. Exp. Allergy 40, 1142-1154 (2010).

35. Chu, H.W. et al. Function and regulation of SPLUNC1 protein in Mycoplasma infection and allergic inflammation. J. Immunol. 179, 3995-4002 (2007).

36. Juhn, Y.J. et al. Increased risk of serious pneumococcal disease in patients with asthma. J. Allergy Clin. Immunol. 122, 719-723 (2008).

37. Bisgaard, H. et al. Childhood asthma after bacterial colonization of the airway in neonates. N. Engl. J. Med. 357, 1487-1495 (2007).

38. Dobrovolskaia, M.A. et al. Induction of in vitro reprogramming by Toll-like receptor (TLR)2 and TLR4 agonists in murine macrophages: effects of TLR "homotolerance" versus "heterotolerance" on NF-kappa B signaling pathway components. J. Immunol. 170, 508-519 (2003).

39. Trompette, A. et al. Allergenicity resulting from functional mimicry of a Toll-like receptor complex protein. Nature (London) 457, 585-588 (2009).

40. Mueller, G.A. et al. The structure of the dust mite allergen Der $p 7$ reveals similarities to innate immune proteins. J. Allergy Clin. Immunol. 125, 909-917 (2010).

41. Matthias, K.A., Roche, A.M., Standish, A.J., Shchepetov, M. \& Weiser, J.N. Neutrophil-toxin interactions promote antigen delivery and mucosal clearance of Streptococcus pneumoniae. J. Immunol. 180, 6246-6254 (2008).

42. Van Den Steen, P.E. et al. Gelatinase B/MMP-9 and neutrophil collagenase/MMP-8 process the chemokines human GCP-2/CXCL6, 


\section{ARTICLES}

ENA-78/CXCL5 and mouse GCP-2/LIX and modulate their physiological activities. Eur. J. Biochem. 270, 3739-3749 (2003).

43. Renckens, R. et al. Matrix metalloproteinase-9 deficiency impairs host defense against abdominal sepsis. J. Immunol. 176, 3735-3741 (2006).

44. Yamada, M. et al. Interferon- \\{gamma $\}$ production by neutrophils during bacterial pneumonia in mice. Am. J. Respir. Crit. Care Med. 183, 1391-1401 (2011).

45. Segal, A.W. How neutrophils kill microbes. Annu. Rev. Immunol. 23, 197-223 (2005)
46. Soehnlein, O., Weber, C. \& Lindbom, L. Neutrophil granule proteins tune monocytic cell function. Trends Immunol. 30, 538-546 (2009).

47. Papayannopoulos, V. \& Zychlinsky, A. NETs: a new strategy for using old weapons. Trends Immunol. 30, 513-521 (2009).

48. Townsend, J.M. et al. IL-9-deficient mice establish fundamental roles for IL-9 in pulmonary mastocytosis and goblet cell hyperplasia but not $\mathrm{T}$ cell development. Immunity 13, 573-583 (2000).

49. Grunig, G. et al. Requirement for IL-13 independently of IL-4 in experimental asthma. Science 282, 2261-2263 (1998). 